\title{
Evidence for the seat of the strain-producing forces
}

\author{
A. G. Galanopocilos
}

Ricevuto il 1 Settembre 1965

Sumarr. - Evidence is presented which favours the theory that the seat of the strain-producing forces is in the low-rigidity layer near the top of the mantle.

Riassunio. - La nota serve a confermare la teoria secondo la quale le forze deformanti vengono localizzate nello strato a bassa rigiditì, prossimo al mantello superiore (sotto la superficie di Mohorovicic).

\section{INTRODUCTION.}

Båth and Duda (1964) in an effort to improve Benioff's method (1951a), being developed for strain release studies, were led to the conclusion that the earthquake volume $V$, identified with the total aftershock volume, increases with magnitude $M$, according to the following equation:

$$
\log V=9.58+1.47 M \text {. }
$$

For the aftershock area $S$, Båth and Duda derived a new equation:

$$
\log S=4.95+1.21 M
$$

which improves a previous relation:

$$
\log S=5.99+1.02 M,
$$

found by Utsu and Seki (1955). Considering that $V=L W H$ and $S=L W$, where $L, \bar{F}$ and $H$ are respectively, the length, the width and the vertical extent of aftershock zone in $\mathrm{cm}$, we may immediately 
derive from the two relations given by Bath and Duda for the earthquake volume and the aftershock zone, the following expression:

$$
\log H=4.63+0.26 M
$$

The equation derived implies that for earthquakes of magnitude 7.7 to 8.7 the vertical extent of aftershock zone reaches to at least 43 to $78 \mathrm{~km}$ depths. Taking this for granted and another very interesting result of Bàth's and Duda's investigation that "The ratio of fault plane area to the vertical section through the aftershock zone, i.e. F/LH, increases with magnitude, approaching unity for the largest shocls", it might be concluded that the middle of the fault plane of the great shocks is at least in most cases - immediately below the Mohorovicic discontimuity, i.e. in the low-rigidity layer near the top of the mantle. This implications is strongly supported by the fact that all shocks of magnitude $>7^{3} / \mathrm{s}$ occurred in the area of Greece are of intermediate focal depth.

The Alaskan earthquake of March 2T, 1964, with Richter magnitude of 8.4 to 8.6 , had a focal depth of about $50 \mathrm{~km}$. In a recent investigation, F. Press (1965) was led to the conclusion " on the basis of the very gradual reduction of vertical deformation with distances that the fault could not have extended to depths as shallow as $50 \mathrm{k}: \mathrm{m}$ and that 100 to $200 \mathrm{~km}$ are more likely values. The fault probably came to within 15 to $20 \mathrm{~km}$ of the surface". Thus the vertical extent found for the fault of the Alaskan earthquake exceeds the values previously found for other earthquakes. According to Press "The larger magnitude associated with the Alaskan earthqualie ( $M=8.4$ ) as compared with the others may partially explain the difference".

The fact that mantle surface waves and free oscillations are excited only by the larger earthquakes strengthens the notion that the primary faulting associated with these shocks extends far below the earth's erust.

Another evidence are the empirical relations of $I_{0}$ to $M I$ derived for constant depths of foci by V. Karnik (1964):

$$
\begin{array}{ll}
h=3-5 \mathrm{~km}, & M=0.56 I_{o}+0.45 \\
h=6-10 \mathrm{~km}, & M=0.56 I_{o}+0.83 \\
h=11-18 \mathrm{~km}, & M=0.57 I_{o}+0.85 \\
h=19-30 \mathrm{~km}, & M=0.50 I_{o}+1.75 \\
h=31-50 \mathrm{~km}, & M=0.40 I_{o}+2.36 \\
h=51-82 \mathrm{~km}, & M=0.29 I_{o}+3.77, M_{L H} \text { without } 2 M^{h} \\
h=83-150 \mathrm{~km}, & M=0.33 I_{0}+3.23
\end{array}
$$


The relations derived for $M=M_{L H}+\partial M^{n}$ without depth corrections, $\partial M^{h}$, have approximately a common intersection near $I_{o}=\mathrm{XI}$ and $M=7$. This implies that in case of shallow shocks the maximum intensity ever observed, $I_{o}=\mathrm{XI}$, is attained by shocks of magnitude 7 ; in other words there are no shallow shocks with magnitude greater than 7 .

\section{DATA USEN.}

In the following table we give all shallow and intermediate earthquakes of magnitude $>5^{1 / 2}$ occurred in the area of Greece during the 120-years interval, 18+3-1962, for which period the available data were proved to be fairly homogenous. In the intermediate earthquakes we have included all earthquakes of focal depth $>40 \mathrm{~km}$.

During the time interval considered there were released: 454 shallow shocks with $M>5^{1 / 2}, 210$ with $M>6,83$ with $M>6^{1 / 2}, 25$ with $M$ $>7$ and 12 with $M>71 / 2$. During the same period, 1843-1962, there were released: 74 intermediate shocks with $M>5^{1 / 2}, 48$ with $M>6$, 39 with $M>6^{1 / 2}, 17$ with $M>7,11$ with $M>71 \%$ and 7 with $M I>8$. The data given above may be summarized as follows:

$\begin{array}{rrrrrrc}M & 51 / 2 & 6 & 61 / 2 & 7 & 71 / 2 & 8 \\ N_{1} & 3.78 & 1.75 & 0.69 & 0.21 & 0.10 & - \\ N_{2} & 0.62 & 0.40 & 0.325 & 0.14 & 0.092 & 0.058 \\ \log N_{1} & 0.58 & 0.24 & -0.16 & -0.68 & -1.00 & - \\ \log N_{2} & -0.21 & -0.40 & -0.49 & -0.85 & -1.04 & -1.23\end{array}$

where $N_{1}, N$. is respectively the number of shallow and intermediate shocks of magnitude $M$ or greater per one year. These values fit rather closely to:

$$
\begin{aligned}
& \log N_{1}=[-1.43+0.82(8-M)] \pm 0.05=-1.43 \pm 0.06+ \\
& +(0.82 \pm 0.02)(8-M) \\
& \log N_{9}=[-1.23+0.42(8-M)] \pm 0.06=-1.23 \pm 0.03+ \\
& \quad+(0.42 \pm 0.01)(8-M) .
\end{aligned}
$$

The logarithm of the ratio of the number of smaller shallow shocks to that of intermediate shocks in the area considered is approximately 3.00 ; this makes the ratio of numbers about 1000: 1 . However, the logarithm of the ratio of the number of shallow shocks of magnitude 8 and over, to that of intermediate shocks is approximately -0.20 ; this makes the ratio of numbers about $0.63: 1$, i.e. roughly $60 \%$ of shocks 
Table I - Catalogue of sinallow martilquakes of magitude $>5^{1 / 2}$

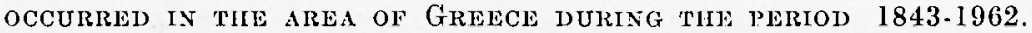

\begin{tabular}{|c|c|c|c|c|c|c|c|c|}
\hline No & & Date & & $\begin{array}{l}\text { Magnitude } \\
\text { adopted }\end{array}$ & No & & Date & $\begin{array}{l}\text { Magnitude } \\
\text { adopted }\end{array}$ \\
\hline 1 & 1843 & Sept. & 5 & $61 / 4$ & 51 & & March 6 & $61 / 4$ \\
\hline 2 & & Oct. & 18 & $63 \%$ & 52 & & March 13 & $51 / 2$ \\
\hline 3 & 1845 , & June & 23 & $61 / 4$ & $5 \overline{3}$ & & March 25 & \\
\hline 4 & & Oct. & 11 & 6.8 & 54 & & Dec. & $63 / 4$ \\
\hline 5 & 1846, & June & 10 & 7.2 & 55 & 1867, & Jall. 27 & $63 \%$ \\
\hline 6 & & June. & 13 & $61 / 4$ & 56 & & March 7 & $6.7^{4}$ \\
\hline 7 & 1848 & - & - & $61 / 4$ & 57 & & March 30 & $61 / 4$ \\
\hline 8 & 1851 , & Jan. & 20 & $61 / 4$ & 58 & & Oct. 22 & 5.9 \\
\hline 9 & & Febr. & 28 & $63 \%$ & 59 & 1868 & Apr. $\quad 20$ & $63 / 4$ \\
\hline 10 & & Oet. & 12 & 7.1 & 60 & & May & $51 / 2$ \\
\hline 11 & & Oct. & 17 & $63 / 4$ & 61 & & Oct. & $5.6^{2}$ \\
\hline 12 & & Dec. & 29 & $61 / 4$ & 62 & 1869, & March 18 & $51 / 2$ \\
\hline 13 & & Dec. & 29 & $61 / 4$ & 63 & & Aug. $\quad 14$ & $63 \%$ \\
\hline 14 & 1852, & Ang. & 26 & $61 / 4$ & 64 & & Sept. & $61 \%$ \\
\hline 15 & 1853, & Aug. & 18 & $7.2^{4}$ & 65 & & Dec. & $7 \mathrm{1} / \mathrm{2}$ \\
\hline 16 & 1854, & July & 30 & 6.6 & 66 & & Dec. 28 & $6.9^{2}$ \\
\hline 17 & 1856 , & Nov. & 13 & $63 / 4$ & 67 & 1870 & Aug. $\quad 1$ & $71 / 2$ \\
\hline 18 & 1858, & Febr. & 21 & $71 / 2$ & 68 & & Sept. 28 & $6.2^{2}$ \\
\hline 19 & & Apr. & 5 & $\begin{array}{ll}6 & 1 / 4\end{array}$ & 69 & 1871 , & Jan. 22 & $61 / 4$ \\
\hline 20 & & Sept. & 20 & $6.9^{4}$ & 70 & & Apr. 8 & $61 \%$ \\
\hline 21 & & Oct. & 10 & 6.8 & 71 & & June & $61 \%$ \\
\hline 22 & 1859 & March & 13 & $61 / 4$ & 72 & & Oct. & 6.9 \\
\hline 23 & & Aug. & 13 & $51 / 2$ & 73 & 1872 , & Febr. 11 & $71 / 2$ \\
\hline 24 & & Aug. & 21 & $6.4^{2}$ & 74 & 1873, & Jan. 31 & $6.6^{2}$ \\
\hline 25 & & Sept. & 12 & $6^{1 / 4}$ & 75 & & July 25 & $61 / 4$ \\
\hline 26 & 1860 & Apr. & 10 & $71 / 2$ & 76 & & Oct. 25 & $6.1^{14}$ \\
\hline 27 & & Apr. & 15 & $51 / 2$ & 77 & 1874 & Jan. 17 & $51 / 2$ \\
\hline 28 & & Apr. & 16 & $61 \%$ & 78 & & March 18 & $51 / 2$ \\
\hline 29 & & May & 16 & $61 / 4$ & 79 & & Nov. 16 & $7.3^{2}$ \\
\hline 30 & & Aug. & 6 & $51 / 2$ & 80 & 1875 & Apr. & $51 / 2$ \\
\hline 31 & 1861, & Dec. & 26 & $71 / 2$ & 81 & & July & $63 \%$ \\
\hline 32 & 1862 , & March & 14 & $71 / 2$ & 82 & 1876 & June 26 & $61 \%$ \\
\hline 33 & & Oct. & 4 & $63^{3 / 2}$ & 83 & 1877 , & July & $51 / 2$ \\
\hline 34 & 1863 & Aug. & - & $61 / 4$ & 84 & & Oct. 13 & $51 / 2$ \\
\hline 35 & 1864 , & June & 14 & $51 / 2$ & 85 & & Oct. & $61 / 4$ \\
\hline 36 & & July & 17 & $51 / 2$ & 86 & 1880, & July & 6.6 \\
\hline 37 & & Aug. & 21 & $5^{1 / 2}$ & 87 & 1881 , & Apr. & 6.3 \\
\hline 38 & 1865 & July & 23 & $6.6^{2}$ & 88 & 1883 , & Oet. & 6.4 \\
\hline 39 & & oct. & 10 & $61 / 4$ & 89 & 1885 , & Febr. 18 & $51 / 2$ \\
\hline 40 & & Oct. & 11 & $51 / 2$ & 90 & & March 28 & $6.0^{2}$ \\
\hline 41 & & Nov. & 11 & $61 / 4$ & 91 & & Dec. 14 & $51 / 2$ \\
\hline 42 & 1866 , & Jan. & 2 & $63 / 4$ & 92 & 1886 & Sept. & $51 / 2$ \\
\hline 43 & & Jan. & 13 & 6.8 & 93 & & Nov. 27 & $51 / 2$ \\
\hline 44 & & Jan. & 31 & 6.1 & 94 & 1887, & May 14 & $6.5^{2}$ \\
\hline 45 & & Febr. & 2 & $61 /$. & 95 & & Oct. & 6.3 \\
\hline 46 & & Febr. & 6 & $\begin{array}{lll}6 & 1 & 1\end{array}$ & 96 & 1888 & Sept. & 5.8 \\
\hline 47 & & Febr. & 28 & $61 \%$ & 97 & 1889 , & Apr. - & $51 / 2$ \\
\hline 48 & & March & 2 & $61 / 4$ & 98 & & Aug. 25 & $6.3^{2}$ \\
\hline 49 & & March & 2 & $63 / 4$ & 99 & & Oct. 25 & 6.9 \\
\hline 50 & & March & 2 & $51 / 3$ & 100 & 1890. & May & $51 / 2$ \\
\hline
\end{tabular}


Table I (cont.)

\begin{tabular}{|c|c|c|c|c|c|c|c|c|c|}
\hline No & & Date & & $\begin{array}{l}\text { Magnitude } \\
\text { adopted }\end{array}$ & No & & Date & & $\begin{array}{l}\text { Magnitude } \\
\text { adopted }\end{array}$ \\
\hline 101 & 1890 & May & 26 & 6.6 & 152 & 1907, & Aug. 1 & 16 & $61 / 4$ \\
\hline 102 & & Dec. & 14 & 6.5 & 153 & 1909 & March & & $5^{1 / 2}$ \\
\hline 103 & 1891 & May 1 & 11 & 6.1 & 154 & & May 3 & & 5.9 \\
\hline 104 & & June 2 & 27 & $51 / 2$ & 155 & & July $\quad$ l & 15 & 5.8 \\
\hline 105 & & Sept. 1 & 18 & $51 / 2$ & 156 & 1910 & Oct. & 27 & $61 / 4$ \\
\hline 106 & 1892 & Jan. & 9 & 5.5 & 157 & 1911, & Febr. 1 & 18 & 7.1 \\
\hline 107 & & Dec. 2 & 27 & $51 / 2$ & 158 & & March & 9 & $51 / 2$ \\
\hline 108 & 1893 & Jan. & 31 & 6.4 & 159 & & Oet. 2 & 22 & $51 / 2$ \\
\hline 109 & & Febr. & 1 & $6^{3} / 4$ & 160 & 1912, & Febr. 1 & 13 & $7.0^{\circ}$ \\
\hline 110 & & Febr. & 9 & 5.9 & 161 & & Febr. 1 & 15 & $61 / 4$ \\
\hline 111 & & March 1 & 12 & $51 / 2$ & 162 & & Apr. & 1 & $51 / 2$ \\
\hline 112 & & Apr. 1 & 17 & 6.4 & 163 & & Apr. 1 & 19 & $51 / 2$ \\
\hline 113 & & May 2 & 23 & 6.1 & 164 & & Apr. 2 & 21 & $53 / 4$ \\
\hline 114 & & June 1 & 14 & $71 / 2$ & 165 & & Aug. & 9 & $73 / 4$ \\
\hline 115 & & Oct. 1 & 13 & $51 / 2$ & 166 & 1913 & May & 25 & $51 / 2$ \\
\hline 116 & 1894 & Apr. & 20 & $6.7^{2}$ & 167 & 1914, & Oct. & 17 & $6.0^{\circ}$ \\
\hline 117 & & Apr. & 27 & 6.9 & 168 & & Oet. l & 17 & 5.9 \\
\hline 118 & & July 2 & 26 & $61 / 4$ & 169 & 1914, & Nov. 2 & 23 & $51 / 2$ \\
\hline 119 & 1895 & May & 13. & $63 / 4$ & 170 & & Nov. 2 & 27 & 6.1 \\
\hline 120 & & May & 14 & $61 / 4$ & 171 & & Dec. & 2 & $51 / 2$ \\
\hline 121 & & May & 14 & $71 / 2$ & 172 & 1915 & Jan. & 27 & 6.0 \\
\hline 122 & & May & 15 & 6.4 & 173 & & June & 4 & 5.7 \\
\hline 123 & & June 2 & 21 & $61 / 4$ & 174 & & Ang. & 7 & 6.3 \\
\hline 124 & & Aug. & 6 & $634_{4}^{4}$ & 175 & & Aug. 1 & 10 & 6.1 \\
\hline 125 & & Aug. 1 & 19 & $\begin{array}{ll}6 & 1 / 4 \\
4\end{array}$ & 176 & & Aug. & 10 & 6.1 \\
\hline 126 & & Sept. & 5 & $61 \%$ & 177 & & Aug. & 11 & 6.4 \\
\hline 127 & 1896 & Febr. 1 & 10 & $5 \frac{1 / 2}{2}$ & 178 & & Aug. I & 11 & 5.5 \\
\hline 128 & & Febr. 1 & 10 & $61 / 4$ & 179 & & Aug. & 19 & 5.7 \\
\hline 129 & & Febr. 1 & 10 & $61 / 4$ & 180 & 1916, & Sept. 2 & 27 & 5.5 \\
\hline 130 & & Febr. 1 & 11 & $51 / 2$ & 181 & 1917, & March 1 & 14 & $61 / 4$ \\
\hline 131 & & Febr. 1 & 11 & $61 \%$ & 182 & & March 2 & 26 & $51 / 2$ \\
\hline 132 & & Nareh 1 & 18 & $61 / 4$ & 183 & & Sept. 2 & 23 & $5 \%$ \\
\hline 133 & & Oct. 2 & 27 & $6.2^{7}$ & 184 & 1918 & Jan. & 17 & $51]_{2}$ \\
\hline 134 & & Nov. 1 & 13 & $51 / 2$ & 185 & & Sept. 1 & 11 & $51 / 2$ \\
\hline 135 & 1897, & Febr. 1 & 12 & $6.1^{12}$ & 186 & & Nov. 2 & 20 & $5 \frac{1 / 2}{2}$ \\
\hline 136 & & June 3 & 30 & $51 / 2$ & 187 & 1919 & Febr. 2 & 24 & 5.7 \\
\hline 137 & 1898 & Febr. 1 & 11 & $51 / 2$ & 188 & & Oct. 2 & 25 & 6.0 \\
\hline 138 & & June & 2 & $6.4^{2}$ & 189 & & Nov. 1 & 18 & $5^{3 / 4}$ \\
\hline 139 & & July & 31 & 6.2 & 190 & 1920 & Oct. & 18 & $61 / 4$ \\
\hline 140 & & Nov. & 9 & 6.0 & 191 & & Oct. 2 & 21 & 5.9 \\
\hline 141 & 1899 & Jan. & 22 & 6.7 & 192 & & Nov. 1 & 15 & 6.1 \\
\hline 142 & & Sept. 2 & 20 & $63 / 4$ & 193 & & Nov. & 25 & $51 / 2$ \\
\hline 143 & 1902 & July & 5 & 6.6 & 194 & & Nov. 2 & 26 & 6.4 \\
\hline 144 & & Nov. & 5 & $51 / 2$ & 195 & & Nov. & 28 & $51 / 2$ \\
\hline 145 & 1904 & Apr. & 4 & $71 / 2$ & 196 & & Nov. 2 & 29 & 6.2 \\
\hline 146 & 1905 & June & 1 & $51 / 2$ & 197 & & Dec. & 18 & $63 / 4$ \\
\hline 147 & & June & 3 & $6.0^{\circ}$ & 198 & & Dec. & 23 & $61 / 4$ \\
\hline 148 & & Oct. & 8 & 6.4 & 199 & 1921 & March & 30 & 5.5 \\
\hline 149 & & Nov. & 8 & 7.0 & 200 & & July & 6 & 5.5 \\
\hline 150 & 1906 & March & 3 & $61 / 4$ & 201 & 1921 & Sept. & 13 & 5.5 \\
\hline $15 \mathrm{l}$ & 1906 & Sept. 2 & 28 & $61 / 4$ & 202 & & Oct. & 21 & $61 / 4$ \\
\hline
\end{tabular}


Table I (cont.)

\begin{tabular}{|c|c|c|c|c|c|c|c|c|c|}
\hline No & & Date & & $\begin{array}{l}\text { Magnitude } \\
\text { adopted }\end{array}$ & No & & I)ate & & $\begin{array}{c}\text { Magnitude } \\
\text { adopted }\end{array}$ \\
\hline 203 & 1922, & Augr. & 11 & $53 / 4$ & 254 & & May & 11 & $61 / 4$ \\
\hline 204 & 1923 & Jan. & 7 & $61 / 4$ & 255 & & Aug. & 17 & $6.3^{3}$ \\
\hline 205 & & May & 6 & $6.3^{1}$ & 256 & 1934 & rebr. & 4 & $51 / 2$ \\
\hline 206 & & Jec. & 5 & 5.7 & 257 & & Febr. & 21 & j $1 / n$ \\
\hline 207 & & Dec. & 27 & $51 / 2$ & 258 & & Nov. & 21 & $53 / 1$ \\
\hline 208 & 1925, & Febr. & 7 & $51 / 2$ & 259 & 1935 & Jan. & 4 & $61 / 4$ \\
\hline 209 & 1926 , & March & 18 & $6.9^{2}$ & 260 & & Jan. & 4 & $5.8^{12}$ \\
\hline 210 & & Mareh & 18 & $51 / 2$ & 261 & & Jan. & 4 & 6.0 \\
\hline 211 & & March & 19 & $51 / 2$ & 262 & & Febr. & 18 & 5.5 \\
\hline 212 & & Dec. & 17 & $6.2^{2}$ & 263 & & March & 30 & 6.0 \\
\hline 213 & & Dec. 1 & 17 & 6.5 & 264 & & $\mathrm{May}$ & 2 & $51 / 2$ \\
\hline 214 & 1927, & Mareh & 24 & $51 / 2$ & 265 & & Nov. & 7 & $6.0^{2}$ \\
\hline $21 \tilde{5}$ & & June & 30 & $5.5^{2}$ & 266 & 1936 & Apr. & 8 & 6.0 \\
\hline 216 & 1928 & Jan. & 23 & 5.5 & 267 & & $\Delta_{p r}$. & 15 & 5.7 \\
\hline 217 & & March & 31 & $61 / 1$ & 268 & 1937, & Jan. & 2 & $51 / 2$ \\
\hline 218 & & Apr. & 18 & $63 / 4$ & 269 & & May & $2 \overline{3}$ & $5.7^{2}$ \\
\hline 219 & & Apr. & 18 & $51 / 2$ & 270 & & July & 6 & 6.0 \\
\hline 220 & & Apr. & 22 & $6.8^{2}$ & 271 & 1938. & Jair. & 30 & 5.9 \\
\hline 221 & & Apr. & 25 & $51 / 2$ & 272 & & Febr. & 10 & 53 \\
\hline 222 & & Apr. $\quad 2$ & 28 & $51 / 2$ & 273 & & March & 11 & 5.8 \\
\hline 223 & & Dee. & 10 & $5.6^{2}$ & 274 & & March & 13 & 5.7 \\
\hline 224 & 1930 , & Jan. & 23 & 5.7 & 275 & & May & 12 & $51 / 2$ \\
\hline 225 & & Jan. & 28 & $63 / 1$ & 276 & & June & 20 & $5.7^{2}$ \\
\hline 226 & & lebr. 2 & 23 & $5.7^{k}$ & 277 & & July & 2 & 5.8 \\
\hline 227 & & March 3 & 31 & 6.1 & 278 & & July & 20 & $5 \mathbf{3}$ \\
\hline 228 & & Apr. & 17 & 5.8 & 279 & 1939 & May & 20 & $51 / 2$ \\
\hline 229 & & June & 25 & 5 i: & 280 & & June & 23 & $51 / 2$ \\
\hline 230 & & Nov. & 21 & $6.0^{2}$ & 281 & & Aug. & 9 & $6.1^{2}$ \\
\hline 231 & & Nov. 2 & 21 & $61 / 4$ & 282 & & Sept. & 22 & $61 / 2$ \\
\hline 232 & & I)ec. & 2 & $63 \%$ & 283 & 1940 & Jall. & 6 & $5.6^{2}$ \\
\hline 233 & 1931, & Jan. & 4 & 5.6 & 284 & & Fehr. & 23 & $51 / 2$ \\
\hline 234 & & March & 7 & 6.0 & 285 & & Fehr. & 29 & $6.0^{2}$ \\
\hline 235 & & Mareh & 8 & $63 / 4$ & 286 & 1941, & March & 1 & $61 / 4$ \\
\hline 236 & & July & 12 & 5.8 & 287 & & May & 14 & $51 / 2$ \\
\hline 237 & 1931, & Sept. 1 & 13 & 6.0 & 288 & & May & 16 & $51 / 2$ \\
\hline 238 & & Nov. 1 & 15 & $51 / 2$ & 289 & & May & 23 & $6.0^{2}$ \\
\hline 239 & & Nov. & 23 & $6.4^{2}$ & 290 & & May & 28 & $51 / 2$ \\
\hline 240 & 1932 & Marceh & 9 & $51 / 2$ & 291 & & June & 24 & $51 / 2$ \\
\hline 241 & & Apr. & 27 & $51 / 2$ & 292 & & July & 13 & $53 / 4$ \\
\hline 242 & & May & 14 & $51 / 2$ & 293 & & July & 23 & $51 / 2$ \\
\hline 243 & & June 2 & 29 & $5 \%$ & 294 & & Sept. & 1 & $51 / 2$ \\
\hline 244 & & Sept. 2 & 26 & $6.9^{2}$ & 295 & & Dec. & 13 & $6.0^{2}$ \\
\hline 245 & & Sejt. 2 & 26 & 6.1 & 296 & 1942 , & Jume & 16 & 5.6 \\
\hline 246 & & Sejt. 2 & 29 & $61 / 4$ & 297 & & Aug. & 27 & $51 / 2$ \\
\hline 247 & & Sept. 3 & 30 & $51 / 2$ & 298 & & Sept. & 1 & $5.8^{\circ}$ \\
\hline 248 & & oct. & 23 & $\begin{array}{ll}5 & 1\end{array}$ & 299 & & Oct. & 28 & 6.0 \\
\hline 249 & & Nov. & 1 & $51 / 2$ & 300 & & Oct. & 28 & 6.0 \\
\hline 250 & 1933 & Jan. & 5 & $51 / 2$ & 301 & 1943 , & July & 23 & $53 / 4$ \\
\hline 251 & & March 1 & 14 & $5.6^{2}$ & 302 & 1944 , & Mareh & 14 & $5.9^{4}$ \\
\hline 252 & & Apr. $\quad 2$ & 28 & $51 / 2$ & 303 & & June & 25 & 6.0 \\
\hline 253 & & May & 8 & 6.1 & 304 & & July & 30 & 5.6 \\
\hline
\end{tabular}


Table I (*out.)

\begin{tabular}{|c|c|c|c|c|c|c|c|c|c|}
\hline No & & Date & & $\begin{array}{l}\text { Magnitude } \\
\text { adopted }\end{array}$ & No & & Date & & $\begin{array}{l}\text { Magnitude } \\
\text { adopted }\end{array}$ \\
\hline 305 & 1944 & ()et. & (6) & 7.2 & 356 & & Aurr. & 12 & 6.2 \\
\hline 306 & & oct. & 7 & 5) $1: 2$ & $35 \%$ & & Aug. & 12 & $51 / 2$ \\
\hline 307 & 1945, & Sept. & 2 & 61 & 358 & & lugr. & $1 \overline{2}$ & $6.0^{2}$ \\
\hline 308 & 1946 & Apr. & 12 & $5 \quad 1 / 2$ & 359 & & lugr. & 12 & 5.5 \\
\hline 309 & & Apr. & 16 & $5.5^{2}$ & 360 & & Augr. & 13 & $51 / 0$ \\
\hline 310 & 1947, & Mareh & 21 & 51 & 361 & & Sept. & 5 & 5.8 \\
\hline 311 & & Apr. & 12 & $5.7^{2}$ & 362 & & Sispt. & 14 & 5.7 \\
\hline 312 & & June & 1 & $51:$ & 363 & & () & 10 & $51 ;$ \\
\hline 313 & & July & 21 & $5.5^{2}$ & 364 & & oct. & 21 & $5.7^{2}$ \\
\hline 314 & & Aug. & 30 & 6.3 & 365 & & oct. & 21 & 6.5 \\
\hline 315 & & Oct. & 6 & 7.0 & 360 & & Nov. & 28 & $51 \%$ \\
\hline 316 & & Nov. & 29 & 5110 & 367 & & Dec. & 28 & $51 / 2$ \\
\hline 317 & & Dec. & 9 & $5.5^{2}$ & 368 & 1954 & Jail. & 2 & $51 \frac{2}{2}$ \\
\hline 318 & 1948 & March & 29 & $51 / 2$ & 369 & & II arcell & & $51 / 2$ \\
\hline 319 & & Ilareh & 29 & $5.9^{2}$ & 370 & & Apr. & 17 & $51 / 2$ \\
\hline 320 & & Apr. & 22 & 6.4 & 371 & & Apr. & 30 & $7.0^{2}$ \\
\hline 321 & & llay & 26 & 5.5 & 372 & & Ilay & 1 & $51 / 2$ \\
\hline 322 & & June & 30 & 6.4 & 373 & 1954, & May" & 3 & 5) 1 \\
\hline 323 & & Oc.t. & 10 & 5.8 & 374 & & Ilay & 4 & $5.7^{2}$ \\
\hline 324 & & Oct. & 18 & 5.5 & 375 & & II ay & 4 & 5.6 \\
\hline 325 & 1949 & Jan. & 4 & 5.7 & 376 & & IIay & 25 & 5.7 \\
\hline 326 & & June & 17 & 6.7 & 375 & & July & 18 & $51 / 2$ \\
\hline 327 & & July & 23 & 6.8 & 378 & & dugr. & 3 & $60^{2}$ \\
\hline 328 & 1950, & Sepit. & 23 & $53 / 4$ & 379 & & Dec. & 23 & 5.9 \\
\hline 329 & 1951 & Apr. & 5 & $6.1^{-1}$ & 380 & 1955 & Jaи. & 3 & 5.8 \\
\hline 330 & & Alur. & 24 & 5.5 & 381 & & March & 28 & $53 / 4$ \\
\hline 331 & & Aur. & 31 & $5^{3} / 4$ & $3 s 2$ & & $A_{p l}$ & 13 & $6.0^{4}$ \\
\hline 332 & & lus. & 31 & 5.6 & 383 & & $\mathrm{Apr}$. & 19 & $6_{1} 11_{1}^{\prime}$ \\
\hline 333 & & oet. & 1 & $51 / 2$ & 384 & & Apr. & 21 & 6.0 \\
\hline 334 & 1952. & March & 19 & $53 / 2$ & 385 & & June & 2 & $51 / 2$ \\
\hline 335 & & oct. & 5 & $5 \quad 3 \quad 4$ & 386 & & July & 9 & $51 / 2$ \\
\hline 336 & & Oct. & 10 & $51 / 2$ & 387 & & $\mathrm{~J}_{\mathrm{ul}}$ & 16 & $6.8^{2}$ \\
\hline 337 & & lot. & 13 & $55^{5 / 2}$ & 388 & 1956, & Jan. & 6 & 5.7 \\
\hline 338 & & Dee. & 31 & $53 / 1$ & 389 & & May & 15 & 5.6 \\
\hline 339 & 1952, & Dece. & 31 & $53 \%$ & 390 & & $\mathrm{May}$ & 15 & 5.8 \\
\hline 340 & l953, & Jall. & 7 & 5.5 & 391 & & May & 18 & 5.8 \\
\hline $3+1$ & & Febr. & 7 & (j. 0 & 392 & & July & 9 & $71 / 2$ \\
\hline 342 & & March & 18 & 7.2 & 393 & & July & 9 & $63 / 4$ \\
\hline 343 & & Mareh & 18 & 5.6 & 394 & & July & 9 & $5.7^{1}$ \\
\hline 344 & & May & 2 & $51 / 2$ & 395 & & July & 9 & 5.6 \\
\hline 345 & & June & 3 & $53 / 2$ & 396 & & July & 9 & $51 / 2$ \\
\hline 346 & & June & 13 & $5 \quad 1 / 2$ & 397 & & July & 9 & $5.5^{2}$ \\
\hline 347 & & June & 18 & 512 & 398 & & July & 10 & $53 / 4$ \\
\hline 348 & & July & 22 & $51 / 2$ & 399 & & July & 10 & $5 \mathrm{3} / 4$ \\
\hline 349 & & Aug. & 9 & $6.5^{2}$ & 400 & & July & 22 & 5.5 \\
\hline 350 & & Aug. & 11 & 6.8 & 401 & & July & 30 & 5.6 \\
\hline 351 & & Aug. & 11 & $51 / 2$ & 402 & & July & 30 & 6.0 \\
\hline 352 & & Aug. & 11 & $51 / 2$ & 403 & & July & 30 & 5.6 \\
\hline 353 & & Aug. & 12 & $5{ }^{5} 1 / 2$ & 404 & & Aug. & 16 & 5.5 \\
\hline 354 & & Alig. & 12 & $7.2^{2}$ & 405 & & Sept. & 6 & 5.7 \\
\hline 355 & & Aug. & 12 & 5.6 & 406 & & Sept. & 16 & $51 / 2$ \\
\hline
\end{tabular}


Table I (cont.)

\begin{tabular}{|c|c|c|c|c|c|c|c|c|c|}
\hline No & & Date & & $\begin{array}{l}\text { Magnitude } \\
\text { adopted }\end{array}$ & No & & Date & & $\begin{array}{l}\text { Magnitude } \\
\text { adopted }\end{array}$ \\
\hline 407 & 1956, & Nov. & 2 & $53 / 4$ & 431 & 1958 & June & 5 & $5^{1 / 2}$ \\
\hline 408 & & Nov. & 20 & $51 / 2$ & 432 & & July & 15 & $51 / 2$ \\
\hline 409 & 1957, & Jan. & 23 & $51 / 2$ & 433 & & July & 17 & $53 / 4$ \\
\hline 410 & & Febr. & 19 & $6.0^{\circ}$ & 434 & & Aug. & 27 & 6.5 \\
\hline 411 & & March & 8 & $61 / 2$ & 435 & & Sept. & 2 & $51 / 2$ \\
\hline 412 & & March & 8 & $63 \%$ & 436 & & Sept. & 4 & $5: 2$ \\
\hline 413 & & March & 8 & $5.6^{\circ}$ & 437 & 1959 & Apr. & 25 & $61 / 4$ \\
\hline 414 & & March & & 6.0 & 438 & & Apr. & 25 & $5.6^{*}$ \\
\hline 415 & & March & 28 & 5.7 & 439 & & May & 14 & $61 / 2$ \\
\hline 416 & & Apr. & 26 & 6.2 & 440 & & June & 10 & $53 / 4$ \\
\hline 417 & & May & 21 & 5.7 & 441 & & Aug. & 16 & $51 / 2$ \\
\hline 418 & & May & 29 & 5.5 & 442 & & Aug. & 17 & $6.0^{\circ}$ \\
\hline 419 & & Oct. & 5 & $51 / 2$ & 443 & & sept. & 1 & $61 / 4$ \\
\hline 420 & & Oct. & 30 & 5.7 & 444 & & Oct. & 5 & $51_{2}^{1 / 2}$ \\
\hline 421 & & Oct. & 30 & $53 / 4$ & 445 & & Oct. & 7 & $53 \%$ \\
\hline 422 & & Nov. & 26 & 5.7 & 446 & & Nov. & 15 & 7.0 \\
\hline 423 & & Nov. & 26 & 5.6 & 447 & & Nov. & 19 & $51 / 2$ \\
\hline 424 & & Nov. & 27 & 5.9 & 448 & & Dec. & I & $53 / 4$ \\
\hline 425 & 1958, & Jan. & 2 & 5.8 & 449 & 1960 & Febr. & 23 & $51 / 2$ \\
\hline 426 & & Jan. & 16 & 5.7 & 450 & & March & 12 & $5.6^{\circ}$ \\
\hline 427 & & March & 15 & 5.6 & 451 & & May & 26 & $61 / 2$ \\
\hline 428 & & Apr. & 3 & $53 / 4$ & 452 & & July & 13 & $51 / 2$ \\
\hline 429 & & May & 3 & $51 / 2$ & 453 & 1961 & Oct. & 2 & $51 / 2$ \\
\hline 430 & & May & 9 & $53 / 4$ & 454 & 1962, & Jan. & 26 & $61 / 4$ \\
\hline
\end{tabular}

of magnitude 8 and over occurred in the area of Greece are of intermediate focal depth. The regression equations for shallow and intermediate shocks define two curves crossing each other at $M=71 / 2$.

Using the data given in Table I and II and applying the relations:

$$
\log E^{1 / 2}=5.9+0.75 M,
$$

and

$$
S \approx \Sigma E^{1 / 2}
$$

where $E$ is the seismic wave energy, $M$ the earthquake magnitude and $S$ the strain release in units $10^{11}(\mathrm{ergs})^{1 / 2}$, we find that the average strain release per shallow and intermediate earthquake, i.e. $S / N$, for the period considered, amounts to 0.4 and 2.1 , respectively. For the 60 -years interval, 1903-1962, the corresponding values are 0.3 and 1.0 . Thus the average strain release per earthquake of intermediate focal depth is 3 to 5 times higher than that per shallow earthquake. 
Table II - Catalogue of earthquakes of MagNitude $>5 \frac{1}{2} 2$ with Focal IEP'TII $\$ 40 \mathrm{KM}$ OCCURRED IN TIE AREA OF GREFCE JUURING TIL PERIOD $1843-1962$.

\begin{tabular}{|c|c|c|c|c|c|c|c|c|}
\hline No & & Wate & $\begin{array}{c}\text { Magnitude } \\
\text { adopted }\end{array}$ & No & & Date & & $\begin{array}{l}\text { Magnitude } \\
\text { adopted }\end{array}$ \\
\hline 1 & 1846 & March 28 & 8.1 & 38 & 1935 & Febr. & 25 & $63 / 4$ \\
\hline 2 & 1856 & Oet. 12 & 8.6 & 39 & & Marcl & 18 & $6 \%$ \\
\hline 3 & 1862, & $J_{\text {une }} 21$ & 6.8 & 40 & 1936 & Apr. & 28 & 53 \\
\hline 4 & 1863 & Apr. 22 & 8.5 & 41 & & Aug. & 8 & 5 \\
\hline 5 & 1867, & Febr. 4 & 7.9 & 42 & 1937, & Dec. & 16 & $61 \%$ \\
\hline 6 & & Sept. 20 & 7.6 & 43 & 1938 & Jan. & 16 & $51 / 2$ \\
\hline 7 & 1869 & Apr. 18 & 6.9 & 44 & & June & 3 & $534^{3}$ \\
\hline 8 & 1886 & Aug. 27 & 8.4 & 45 & & Sept. & 18 & $61 / 2$ \\
\hline 9 & 1887, & July 17 & 7.7 & 46 & 1939 & Sept. & 20 & $61 / 2$ \\
\hline 10 & 1897 & May & 7.6 & 47 & 1942, & May & 9 & $5 \frac{3 \%}{4}$ \\
\hline 11 & 1903 & Alug. 11 & 8.3 & 48 & & May & 21 & $51 / 2$ \\
\hline 12 & 1904 & Aug. 11 & 8.0 & 49 & & June & 21 & $61 \%$ \\
\hline 13 & 1908 & May 17 & $63 / 4$ & 50 & 1943 & Jan. & 7 & $51 / 2$ \\
\hline 14 & 1910 & Febr. 18 & 70 & 51 & & Febr. & 14 & 60 \\
\hline 15 & & Aug. 21 & $61 / 2$ & 52 & & June & 27 & $53 / 4$ \\
\hline 16 & 1911, & Apr. $\quad 4$ & $7.0^{2}$ & 53 & & Oct. & 16 & $61 \%$ \\
\hline 17 & 1912, & Jan. 24 & $63 / 4$ & 54 & 1944, & Jan. & 5 & $5.7^{4}$ \\
\hline 18 & 1913, & Sept. 30 & $\begin{array}{lll}53 / 4 \\
3\end{array}$ & 55 & & May & 27 & $61 / 4$ \\
\hline 19 & 1918 & July 16 & $611_{2}^{4}$ & 56 & & Aug. & 9 & $51 / 2$ \\
\hline 20 & 1922 & Aug. 13 & $6 \frac{12}{3 / 4}$ & 57 & 1946 , & Apr. & 5 & $6.0^{2}$ \\
\hline 21 & & Nov. 11 & $5.7^{2}$ & 58 & 1947, & June & 4 & 60 \\
\hline 22 & 1923 & Aug. & 6.7 & 59 & & July & 7 & $51 / 2$ \\
\hline 23 & 1925 , & July & $61 / 2$ & 60 & 1948 & Febr. & 9 & 7.1 \\
\hline 24 & 1926 & June 26 & $8.3^{2}$ & 61 & & July & 24 & $61 / 2$ \\
\hline 25 & & July 5 & $51 / 2$ & 62 & & Sept. & 11 & 6.5 \\
\hline 26 & & Aug. 30 & $7.0^{2}$ & 63 & 1952, & Der. & 17 & 63 \\
\hline 27 & & Sept. 19 & $61 / 1$ & 64 & 1953， & Febr. & 14 & $53 / 4$ \\
\hline 28 & 1927, & July 1 & $6.0^{4}$ & 65 & & June & 23 & $53 / 4$ \\
\hline 29 & 1929, & March 27 & $53 / 4$ & 66 & 1957. & Apr. & 24 & 70 \\
\hline 30 & & Nov. Il & $5.7^{2}$ & 67 & & Apr. & 25 & $71 / 4$ \\
\hline 31 & 1930, & Febr. 14 & $63 / 4$ & 68 & 1958 & June & 30 & $6 \mathrm{1} / \mathrm{s}$ \\
\hline 32 & & Mareh 6 & $53 / \mathrm{s}$ & 69 & & May & 27 & 5.6 \\
\hline 33 & & March 6 & 6.0 & 70 & & Nov. & 15 & $53 / 4$ \\
\hline 34 & 1931, & June 30 & $5^{1 / 2}$ & 71 & 1960 & Nov. & 4 & 5.8 \\
\hline 35 & 1932, & Aug. $\quad 15$ & $51 / 2$ & 72 & & Nov. & 11 & 5.7 \\
\hline 36 & 1933, & Apr. 23 & $63 / 4$ & 73 & 1961 & Mav & 23 & $61 / 2$ \\
\hline 37 & 1934, & Nov. 9 & $63 / 4$ & 74 & & Nov. & 28 & $51 / 2$ \\
\hline
\end{tabular}

\section{Discussion.}

Assuming with Bath and Duda (1964) that "the main difference between large and small earthqualies is not to be found in the strain but 
in the total volumes involved", it might be possible to think that the higher average strain release per intermediate earthquake is due to the larger ability of deformation of the low-rigidity layer of the upper mantle. However, considering that a large amount of deformation in weak layers is relieved by plastic flow, i.e. that the possibilities of strain storage in the upper mantle are not very good (Baith and Duda, 1963/ITI), the pronounced maximum of average strain release per earthquake at 50 to $150 \mathrm{~km}$ focal depths might be due to a combination of stronger strain accumulation and greater seismic gain ratio. The greater "seismic gain ratio", i.e. the greater ratio between seismic energy and elastic strain energy in the upper mantle, is evidenced by the very small number of aftershocks. Owing to the lower breaking strength of the lowvelocity layer of the upper mantle the strain accumulated in a very large volume is probably completely relieved in almost one earthquake. A stronger stadin accumulation at 50 to $150 \mathrm{~km}$ depths combined with a smaller ability of strain storage implies that the seat of the strain producing forces is in the upper mantle. It is reasoned therefore that convection currents are probably the prevailing agent in producing a strained region in the Earth's interior. This evidence is fairly corroborated by the fact that in Greece the periods of greater seismic activity are initiated by intermediate shocks, i.e. the seismic activity in the area of Greece is induced by processes oceurring under the Farth's crust (Galanopoulos, 1956).

Later on Blot (1963) was led to the same conclusion for other regions of the globe where intermediate and deep foci exist: Southern Pacific, America, Japan, Indonesia, Indo-Kush ... According to Blot "The earthquakes of very great magnitude are preccded by deep earthquales of a magnitude superior to 7 , or by several deep earthquakes of a lesser magnitude".

Recently L. Don Leet and Florence J. Leet (1965), based on other data were led with a difterent reasoning to believe that "the fundamental cause of earthquakes is movement in the soliqueous mantle", and even more that " when compututional procedures are further refined, it may turn out that all earthqualies are basically mantle raptures, oven when some displacements are coupled to the surface".

Benioff (1951b) has already put forward evidence that earthquakes of magnitude exceeding 8, may not be entirely independent events, but may be related in some form of global stress-system. This suggestion is very well understood in the assumption that the seat of the strain producing forces is in the upper mantle. 


\section{ACKNOWLEDGMENTS.}

The research reported in this document has been sponsored by the " $\Lambda$ ir Foree Office of Scientific Research" under contract $\Lambda F$ 61(052)-803 through the "European Office of Aerospace Research (OAR) ", "United States $\Lambda$ ir Force ", as part of the Advanced Research Projects Agency's Project Vela Uniform.

The anthor would like to express his sincere thanks to Dr. B. C. Papazachos for reading critically the manuscript of this paper.

\section{REFERENCES}

BATri M. and DU1) S. J., Earlhqualie Volume, Faull Plane Area, Seismic Energy, Strain, Deformalion and Relaled quanlities, "Ammali di Geofisica ", 17, 353-368, (1964).

BRtir M. and Duda S. J., Strain Release in Relation to Focal Depth, "Geofisica Pura e Applicata ", 56, 93-100, (1963/III).

Beniofr F., Earlhqualies and Rock Creep, Part I: Creep Characteristics of Rocks and the Origin of Aftershocks, "Bull. Seism. Soc. Amer.", 41, 31-62, (1951a).

BeniofF II., Global Strain Accumulation and Release as Revealed by Great Earlhquakes, "Bull. Geol. Soc. Am.", 62, 331-338, (1951b).

Brot C., Deep Origin of Shallow Earlhquales and Tolcanic Eruptions. Abstract submitted to the IUGG General Assembly in Berkoley, 19-31, August 1963.

Don Leet J. and Fonence J. Jeet, The Earth's Mantle, "Bull. Seism. Soc. Am.", 55, 3, 619-625, (1965).

Galanopoulos A., The Seismic Efficiency of Greece, "Prakt. of Athens Academy" 31, 368-375, (1956).

Karnik V., Seismicily of Europe. Progress Report IV prepared for the conference of the European Seismological Commission in September 1964. Mimeographed, 1-22 (Tolio, 1964).

Press F., Displacements, Strains, and Tilts at Teleseismic Dislances. "Jour. Geoph. Res.", 70, 10, 2395-2412, (1965).

Utsu T. and SEni A., Relation between the Area of the Aftershock Region and the Energy of the Main Shock, "Jour. Seism. Soc. Japan", Ser. II, 7, 233-240, (1955). 